Recibido: junio de 2014

Aprobado: agosto de 2014

\section{STORIA RECENTE, USO, DEGRADO E RESTAURO DELLE SCUOLE NAZIONALI D'ARTE DI CUBANACÁN (1999-20I4)*}

Michele Paradiso** - Dipartimento di Architettura, DiDA Università degli Studi di Firenze, Italia
Nuova garitta all'ingresso della Scuola di Danza.

Fonte: Arch. Universo Garcì Lorenzo.

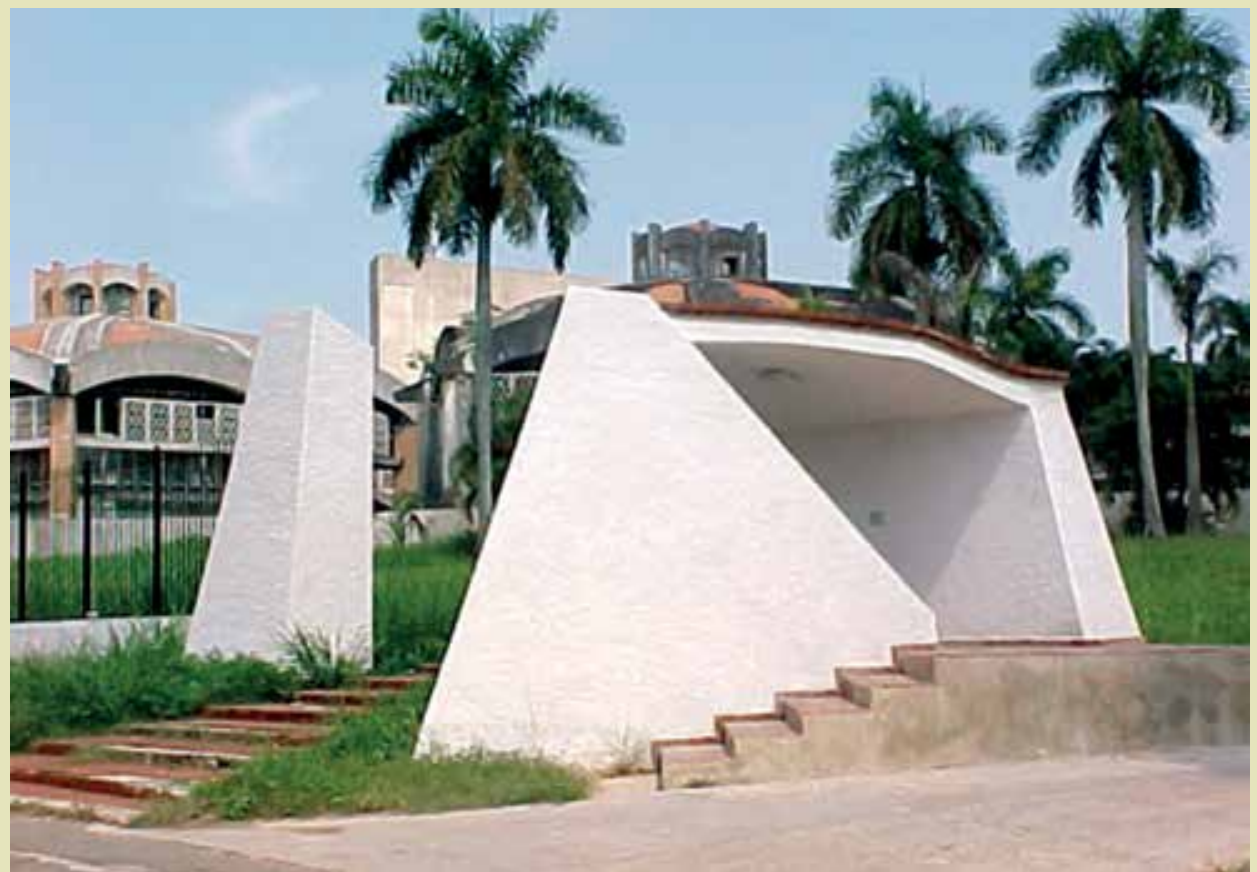

\section{ABSTRACT}

L'articolo racconta la storia recente degli ultimi I 5 anni del restauro e rifunzionalizzazione delle Scuole Nazionali d'Arte, considerate il più bell'esempio dell'architettura post-rivoluzionaria in Cuba. Le Scuole, progettate nel 196I dagli architetti italiani Roberto Gottardi e Vittorio Garatti, e dal cubano Ricardo Porro, hanno avuto una vita molto travagliata, a cominciare dal fatto che la loro realizzazione fu fermata, dopo i fatti di Playa Giron (1964), per diversi motivi. Utilizzate per 40 anni in modo improprio, furono oggetto di un tentativo di restauro, a partire dal 1999.

In questo articolo si racconta la storia di questo tentativo, raccontandone lo stato di degrado alle quali erano giunte, e le vicende che hanno interessato diversi protagonisti, primi fra tutti i tre grandi architetti, tutt'ora viventi e attivi.

\section{PAROLE CHIAVE}

Scuole Nazionali d'Arte, Restauro, Storia 


\section{TESTIMONIO RECIENTE, USO, DETERIORO Y RESTAURACIÓN DE LAS ESCUELAS NACIONALES DE ARTE DE CUBANACÁN (1999-2014)}

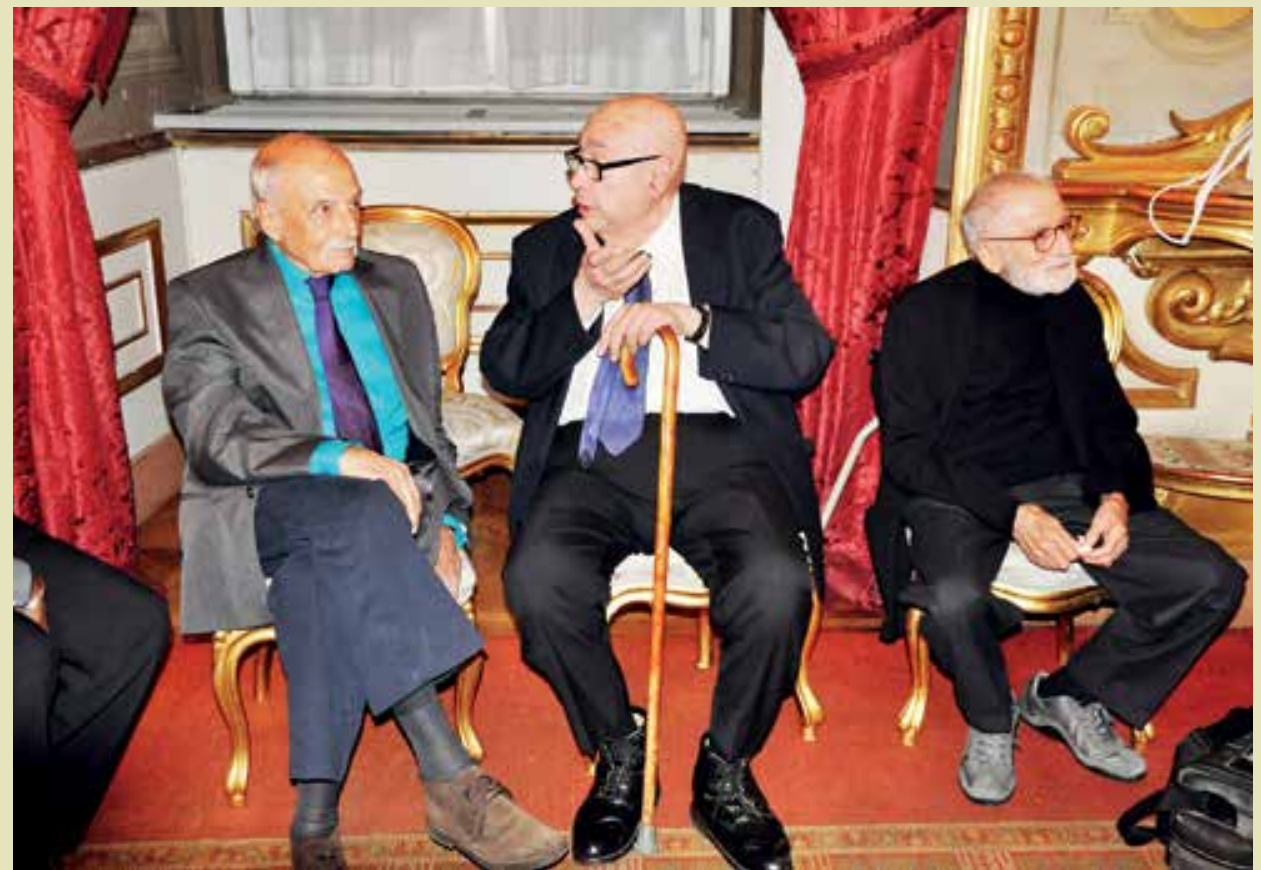

\section{RESUMEN}

El artículo narra los acontecimientos de los últimos 15 años de restauración y reutilización de las Escuelas Nacionales de Arte, las cuales se consideran el ejemplo más bello de la arquitectura posrevolucionaria en Cuba. Las Escuelas, diseñadas en $196 \mathrm{I}$ por los arquitectos italianos Roberto Gottardi y Vittorio Garatti y el cubano Ricardo Porro, han tenido una vida marcada por muchos problemas que inician desde que su construcción fue detenida, por diferentes razones, después de los eventos en Playa Girón (1964). Utilizadas desde hace 40 años en forma inadecuada, han sido objeto de un intento de restauración iniciado en 1999.

En el presente artículo se relata la historia de este intento y describe el estado de degradación al que las Escuelas habían llegado, así como los acontecimientos que han afectado a diversos protagonistas, sobre todo a sus tres grandes arquitectos, todavía vivos y diligentes.

\section{PALABRAS CLAVE}

Escuelas Nacionales de Arte, Restauración, Historia.
Gli architetti italiani Roberto Gottardi (sinistra) e Vittorio Garatti (destra) e el architetto cubano Ricardo Porro (centro) Fonte: Michele Paradiso, 2012
** Profesor de Resistencia de Materiales y de Recuperación Estructural de Monumentos Históricos. Lleva 40 años investigando sobre el comportamiento estructural de arcos y bóvedas de mampostería, y además en técnicas sustentables de consolidación de monumentos históricos. Conferencista y autor de libros, cuenta con más de I 10 publicaciones científicas entre congresos nacionales e internacionales y artículos en revistas nacionales internacionales. Profesor referente de Convenios de Colaboración Académica con diferentes universidades e instituciones extranjeras. Asesor referente del programa de Naciones Unidas PDHL/UNDP. Miembro de Icomos/ Icofort, se ha dedicado a la investigación de las técnicas constructivas de las fortificaciones militares en América Latina. En noviembre de 2013 la Alcaldia de Barichara (Santander), por decreto, le otorgó el título de "Huésped llustre de la Ciudad". 


\section{PREMESSA}

Le vicissitudini architettoniche, tecniche e umane che hanno accompagnato la vita di mesto insieme architettonico, tanto importante nella storia di Cuba e non solo, sono ben raccontate dal libro di John Loomis, Revolution of forms. John Loomis ha avuto l'enorme merito di richiamare l'attenzione della comunità culturale internazionale sullo stato di degrado al quale erano giunte Le Scuole, dopo quasi 40 anni dalla loro idea, che la leggenda vuole essere legata a una partita di golf, con Fidel Castro ed Ernesto Che Guevara, sul tappeto verde del Country Club de La Habana, in qualche modo considerato simbolo del regime del dittatore Batista.

Non staremo e ripetere quello che già John Loomis ha scritto, a cui si sono successivamente riferite quasi tutte le altre iniziative successive, a partire dall'anno 2000, come seminari, articoli su riviste, libri, conferenze, etc. Intendiamo, invece, dare informazioni sulla storia recente del tentativo di restauro, enfatizzando di questo restauro gli aspetti tecnici, che, invece, quasi mai sono stati raccontati, e che, a parere dello scrivente, costituiscono una ulteriore chiave di lettura per intendere il perché si è arrivati alla situazione attuale.

\section{NOTE CRITICHE SULLA TECNICA COSTRUTTIVA DE LAS ESCUELAS}

Prima di entrare nel merito delle diffuse e complesse patologie di degrado che Le Scuole presentavano al momento che se ne decise il restauro, vale la pena di fare alcune considerazioni critiche sulla tecnica costruttiva delle Scuole. John Loomis riporta nel suo testo ed è altrettanto universalmente riconosciuto, che Las Escuelas de Arte de Cubanacàn furono realizzate con una tecnica costruttiva che permettesse, agli albori dell'epoca post-rivoluzionaria cubana, e in una situazione di grave crisi economica del Paese, di economizzare in termini di materiali utilizzati e sofisticatezze tecnico-esecutive. La soluzione fu individuata nella tecnica delle volte catalane, la cui origine si fa risalire in terra spagnola nella zona tra Valencia e Barcellona.

La necessità di risparmiare materiali per costruire fa parte della storia dell'essere umano: $\mathrm{i}$ costruttori di tutti i tempi hanno cercato di eliminare o risparmiarsi la centina, per motivi economici. I Bizantini furono i maestri della costruzione senza centina, in base a studi condotti da Choisy alla fine del XIX secolo, affermando che la volta senza centina è una tecnica romana nella quale veniva utilizzata la cassaforma a perderé per costruire volte apparenti in calcestruzzo. Juan Bergós, invece, individua le origini della tecnica nella cultura costruttiva popolare medievale poiché non si conosce alcun esempio prima del I 400 .

La costruzione senza centina si espande in tutto il Mediterraneo ed è stata utilizzata fino a poco tempo fa nel Nord di Africa e la Spagna, in particolare in Extremadura e Catalogna. Chiamata anche volta catalana per la diffusione del sistema in Catalogna, durante il XIX secolo (architettura catalana modernista: Gaudí, Domenech i Montaner, Puig i Cadafalch, Muncunill, Guastavino e altri), anche se volte di questo genere già apparse alla fine del periodo gotico. Secondo Joan Bassegoda Nonell, le volte senza centina in Catalogna sono posteriori rispetto a quelle di Valencia. Egli sostiene che il primo esempio compare, tra 1310 e 1320, nella Stanza Capitolare del Convento Domenicano (Sala de las Palmeras) di Valencia.

In ogni caso, la straordinaria storia delle volte senza centina, con le sue vicende internazionali, evolve fino al decennio tra 1940 e 1950, periodo in cui, dopo una durata di diversi secoli, l'uso di queste volte vede praticamente la sua fine, eccetto per la costruzione di scale di 
pianta rettangolare ricoperte e sostenute per lo più con volte a botte inclinate, pratica che prevale fino al 1980. Attualmente questa tecnica costruttiva è scomparsa in Spagna. Gli ultimi muratori sono già in pensione. Una "contaminazione" interessante di questa tecnica è attribuita all'uruguaiano Eladio Dieste, che nel incorporare acciaio nei mattoni, scopre un nuovo potenziale estetico di questa tecnica.

È opportuno, a questo punto, richiamare le caratteristiche principali di questa tecnica costruttiva, rimandando gli approfondimenti alla vasta letteratura tecnica disponibile internazionalmente sull'argomento.

La volta catalana è composta di mattoni adagiati orizzontalmente sulla faccia di maggior superficie e sovrapposti in diversi strati, seguendo una curvatura dettata dall'intradosso. Per garantire la sua forma si collocano file di mattoni sottili forati, attaccati con malta di gesso. II mattone ha dimensioni di 300x।50x I5 mm (peso: 0, I2 N/ mm2). Per la escussione si dispongo tre o quattro strati (o più) con malta di gesso e malta cementizia o di calce. II primo strato è posto in modo semplice senza cassaforma con pasta di gesso, ottenendosi la forma grazie alla leggerezza del mattone e alla velocità dell'indurimento . Gli strati seguenti sono disposti in filate doppie, con cemento o calce.

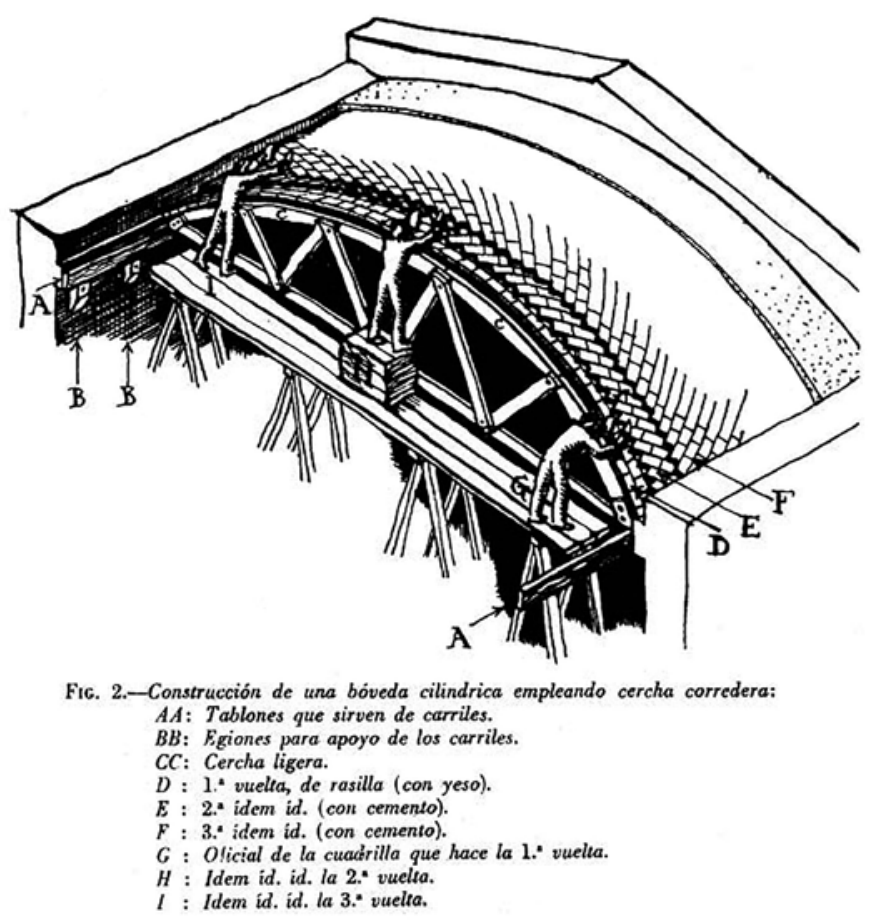

Figura I. Costruzione di una volta cilindrica con capriata scorrevole. Fonte: Prof. Ing. Begonia Serrano Lanzarote, UPV.

Le regole di base per una buona applicazione sono:

- Spostamento relativo tra le unioni dei diversi strati, finimento delle giunte

- Quantità di malta nelle unioni, a rapporto con lo spessore del mattone tra 0,5 e I. Per tre strati $70 \mathrm{~mm}$ e massimo tra 100 e $110 \mathrm{~mm}$ per quattro strati.

- Si fanno allo steso tempo le parti semplici e gli incurvamenti, con un team di persone per ogni strato, lasciando un intervallo tra gli strati. 
Le volte catalane, o di guscio, possono essere classificate a seconda della superficie, della forma e della regione.

Secondo la sua superficie: superficie continua in tutta la volta o superficie divisa con nervature e vele (spicchi della volta a crociera).

Secondo la forma o tipo della volta: volta cilindrica (a botte), volta a intersezione, di crociera, angolari nei chiostri, volta a lunetta, volta a calotta o cupola, volte delle scale

Per regione: Catalogna, Estremadura, Altre.

I vantaggi di questa tecnica si riassumono nella grande economia ottenuta nella messa in opera (senza centina o soltanto uno stampo guida), nella leggerezza in relazione alla sua capacità di carico, nella riduzione delle masse sulle staffe per avere spinte laterali minori, e, infine, nella capacità illimitata di generare forme, come le "Scuole Nazionali d'Arte" ci hanno insegnato.

La scelta di realizzare le Scuole con la tecnica delle volte catalane, utilizzando poco legname per le casseforme, venne in un momento e in una epoca nella quale il cemento armato era il "materiale" principe, soprattutto in America Latina, per le influenze della madre culturale Spagna. Il cemento armato, alla epoca della costruzione delle Scuole, imperava dappertutto, e malgrado esistessero in Cuba e soprattutto a La Habana, esempi di costruzioni in volte catalane, anche a causa degli accadimenti politici della rivoluzione castrista, se ne era persa la sapienza costruttiva.

Di questi edifici rimangono ancora tracce, e sarebbe interessante poterne eseguire una mappatura di quelli che rimangono e del loro precario stato di conservazione. Cionondimeno esistevano precedenti noti, come lo stesso Cabaret Tropicana, che i maligni dicono avesse ispirato Vittorio Garatti per il pasillo di ingresso della sua Scuola di Balletto, o il Club Nautico, ma anche alcuni edifici per civile abitazione, come la Casa Eugenio Leal, in Miramar, del 1957, o la Casa Manuel Gutierrez, in Nuovo Vedado, del 1955.

Tornando alle Scuole, se si esamino le foto storiche della fase costruttiva, e si analizzano nel dettaglio i vari elementi costruttivi, emerge che nelle Scuole abbonda la presenza del cemento armato, rispetto alle zone voltate in càscara, per la presenza, per esempio, dei potenti cordoli di appoggio delle volte e dei corridoi di distribuzione, per l'eccessivo numero di nervature nelle quali le volte furono ripartite, e altro ancora. Già questa osservazione porterebbe portare a definire Las Escuelas de Arte de Cubanacàn, un esempio non proprio filologicamente corretto di volte catalane, piuttosto il risultato di una tecnica mista. Non sembra, allo scrivente, che ciò possa essere dipeso solo dalla notevole luce che cupole e volte dovessero coprire, ma da qualche altra ragione in più. Non altrimenti si spiegherebbe la presenza diffusa, in tutti gli ambienti voltati, a parte la Scuola di Arte Plastica di Ricardo Porro, di tiranti in acciaio ad aderenza migliorata. Per non parlare del fatto che le zone voltate alla catalana presentano nello spessore addirittura nove "capas de resillas"

La storia costruttiva delle Scuole è perfettamente raccontata da John Loomis, ed è noto che il MICONS affiancò ad ognuno dei tre architetti un ingegnere strutturista, che, a parte l'uso delle volte catalane, doveva rendere l'insieme elegante e formalmente libero delle Scuole, strutturalmente sicuro. Viene il sospetto cioè che gli ingegneri strutturisti poco si fidassero di quelle volte catalane di così grande luce e quindi avessero corroborato il progetto di una buona dose di cemento armato. 

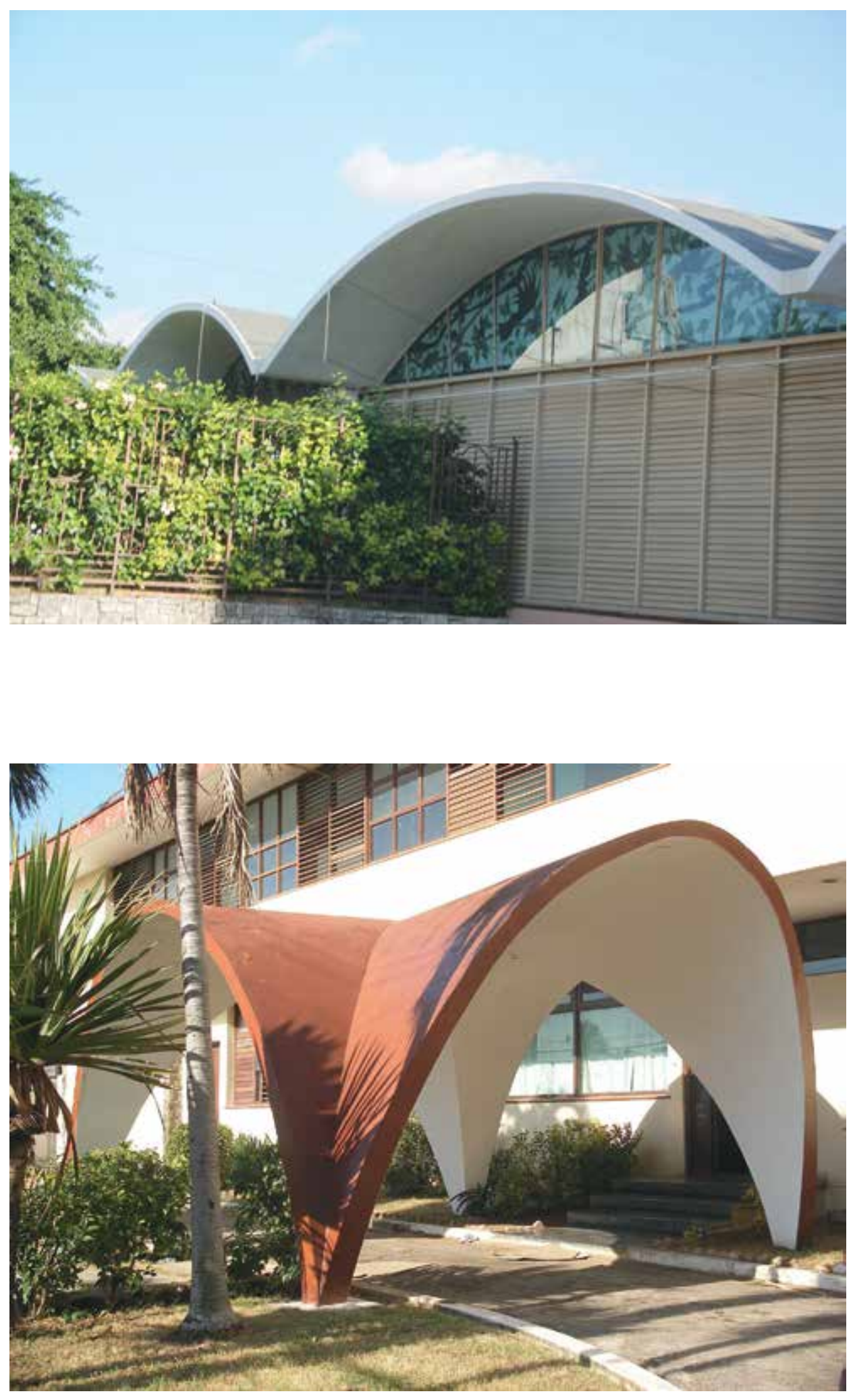

Figura 2. Villa Manuel Gutiérrez. Fonte: Arch. Micol Simoni

Figura 3. Villa Eugenio Leal. Fonte: Arch. Micol Simoni. 
II sospetto fu confermato e, per l'autore di questo articolo, divenne convinzione certa, in una riunione cui lo scrivente partecipò, nella sede del MICONS, nel giugno del 2008. L'Arch. Josefina Rebellòna Alonso, funzionaria di quel Ministero, ma già attiva collaboratrice all'epoca delle Scuole, organizzò un incontro con alcuni dei protagonisti tecnici della esecuzione delle Scuole. Tra questi alcuni ingegneri dell'epoca, nonché l'Arch. José Mosquera e l'Arch. Regino A. Gayoso Blanco. Essi mi confermarono che la complessità delle forme architettoniche, proposte da Porro, Garatti e Gottardi, impose un lavoro di equipe, volto ad assicurare stabilità strutturale al complesso, cosa che, secondo loro, solo il cemento armato poteva fornire, anche perché la natura del terreno, soprattutto nella zona vicino al Rio Quibù, non dava le garanzie necessarie anche in termini di sistema fondale. Alcune delle aule di Garatti, per esempio, poggiano su enormi e spesse platee di fondazioni, come complesso era il sistema fondale dei pasillos di Arte Plastica. Tra gli ingegneri strutturisti che furono affiancati ai progettisti si ricordano tra gli altri Edoardo Esenarro e Isabelita Wittmarch, che furono affiancati a Vittorio Garatti, e llda Fernandez, che collaborava con Ricardo Porro. Emerse anche, in quella riunione, e questo anche contraddicendo quell'idea di avventuriera improvvisazione progettuale che viene in qualche modo riportata dai racconti in prima persona anche dei tre progettisti e poi immancabilmente riportata, con poca verifica documentale, nei vari testi e articoli che si sono scritti, a partire dal 2002, che le Scuole d'Arte furono per il Governo Cubano una occasione di sperimentazione, per arrivare a tipologie costruttive standardizzate che potessero essere disponibili sul mercato edilizio nel futuro, a partire dall'esempio prototipale originario. II MICONS lavorava molto in quegli anni, per le evidenti necessità della epoca postrivoluzionaria, nei sistemi prefabbricati in cemento armato per scuole e viviendas. Ma intuì che se le Scuole avessero raggiunto quei requisiti di funzionalità, sicurezza ed economicità, imposti al progetto, sarebbero potute diventare utili al Paese in future applicazioni. Si lavorò, secondo una sorprendente strategia ministeriale, su tre fronti:

- Ricupero della saggezza costruttiva: si chiamarono all'opera alcuni esperti costruttori di volte catalane per formare muratori capaci in questo tipo di costruzione. Fra questi esperti spiccava la figura dell'ormai ben noto Gumersindo e di un costruttore che faceva di cognome Vijil, entrambi catalani di Valencia. Essi non solo prepararono un programma di apprendistato per i muratori, ma collaborarono attivamente con i tre architetti per disegnare le centine per la costruzione delle volte delle Scuole;

- Industrializzare la costruzione delle volte catalane: si intendeva cioè studiare la possibilità che potessero diventare ripetibili, attraverso le tecniche di prefabbricazione. Fu creato un apposito Dipartimento del Micons, con una propria Direzione di Investigazione Tecnica, il quale dipartimento come prima cosa costruì un Poligono Sperimentale, dove si facevano sperimentazioni e prove di carico su modelli in scala e prove al vero di volte di piccole dimensioni, sia per le Scuole, che per i futuri progetti di prefabbricazione. Uno dei primi Direttori di questo speciale Dipartimento fu l'Arch. Ugo Da Costa Callido. Da Costa fu progettista di molti prototipi sperimentali di elementi prefabbricati in volte catalane, anche a doppia curvatura. Questa esperienza durò dal I96 I al 1965.

- Appoggiare in modo incondizionato il progetto e la costruzione de Las Escuelas de Arte: non si lesinarono a Porro, Gottardi e Garatti, proprio per gli sviluppi che ne sarebbero potuto derivare per il futuro dell'edilizia cubana, né assistenza tecnica, né manovalanza, malgrado il MICONS fosse per necessità impegnato in altre priorità, quali nuove viviendas e scuole, attraverso la filosofia della prefabbricazione. Tra ingegneri, architetti, capicantiere, maestri d'opera e operai, il MICONS mise a disposizione del progetto più di 2000 persone. 
Come si può facilmente notare, questa storia, raccontata da coloro che allora erano parte della strategia del Ministero, fornisce una visione più complessa e articolata della realizzazione delle Scuole, facendone emergere le idee di investimento che il Governo Cubano vedeva in quella operazione. E qui val la pena sottolineare che forse queste figure professionali, solo apparentemente di secondo piano, ma spesso dimenticate dalla Storia dell'Architettura, quali strutturisti, maestri d'opera, semplici operai, andrebbero rivalutate e maggiormente studiate. Fare architettura non è solo pensiero, ma anche azione, e nella azione hanno tutti la stessa importanza. Basta che un maestro concertista stoni, nel mezzo di una esecuzione musicale, perché si perda la manifestazione dell'arte.

\section{CRONACA DI UN RESTAURO: PRIMA PARTE}

I fatti raccontati nella parte sesta della seconda edizione del volume di John Loomis, riferibili alla creazione dei presupposti culturali per la necessità del restauro delle Scuole d'Arte, si fermano alla fine degli anni ' 90 del secolo scorso. In questi ultimi 15 anni molto altro è successo, è stato scritto e detto sull'architettura più significativa della cuba post-rivoluzionaria. Certamente, come riferisce John Loomis, l'ambiente della cultura cubana considerava il restauro delle Scuole una necessità improrogabile, ma mancava ancora la volontà politica a riguardo, anche se già nel 1997 il complesso architettonico era stato dichiarato, dalla Comisión Nacional de Monumentos, Area Protetta (Zona de Protección) ed era stato inserito nell'elenco delle opere emblematiche del Movimento dell'Architettura Moderna a Cuba del DO.CO.MO.MO. Cuba. È opinione dello scrivente, però, che fu determinante a sbloccare la situazione in via definitiva, l'inserimento, proprio in quegli anni, delle Scuole nella lista dei monumenti a rischio del World Monument Fund, grazie all'appoggio di Norma Barbacci, e alla visibilità internazionale che la prima edizione del libro di Loomis aveva dato alle Scuole. Sta di fatto che nel 1999 lo Stato Cubano dà il via ufficialmente alle opere para su "rehabilitación y completamiento". Si badi bene che l'espressione "riabilitazione e completamento" non deve intendersi riferita solamente alla necessità che il complesso architettonico degli anni ' 60 , mai completato, e, per ovvia necessità, utilizzato in modo improprio, necessitasse, in primis, di un restauro e di un completamento dell'opera interrotta, ma a qualcosa di più imponente. Infatti il Governo Cubano approfittò dell'opportunità del restauro per porre mano a una importante riforma didattica, decidendo di affiancare e di far coesistere, alle originarie Escuelas Nacionales de Arte, di formazione pre-universitaria, il nuovo Instituto Superior de Arte, di formazione universitaria, con vere e proprie facoltà dedicate ai differenti campi artistici, senza escludere le forme d'arte più moderne, quali quelle proprie del mondo della fotografia e della multimedialità.

Questo fatto, a parere dello scrivente, scatenerà una polemica nella polemica, quando si andrà a "ripensare" gli edifici di Porro, Garatti e Gottardi, in funzione delle nuove funzioni multiformazione. E sarà la causa di molte incomprensioni e di alcuni determinanti insuccessi.

Tornando alla cronistoria del restauro, il Governo Cubano affidò il progetto di restauro al Ministerio de la Construcción (MICONS), al Ministerio de Cultura (MINCULT) e al Ministerio de Educaciòn Superior (MES). II MICONS utilizzò per l'obiettivo la sua impresa EMPROY n². A coordinare i lavori furono chiamati l'Arch. Universo Garcia Lorenzo, come Proyectista General, e l'Ing. Alejandro Pascual Jorge, come Proyectista Estructural, dello stesso MICONS. Come Asesora Principal de Restauración fu nominata la Dott.ssa Arch. Lucrecia Pérez Echázabal, della Facoltà di Architettura del ISPJAE de La Habana. La parte diagnostica fu affidata al Centro de Investigaciones de las Estructuras y los Materiales (CIDEM), della Universidad Central de las Villas, di fatto coordinato dal Prof. Ing. Gilberto Quevedo Sotolongo. 


\section{ELEMENTI FONDAMENTALI DELL'INTERVENTO TECNICO DI RESTAURO}

Fu inizialmente preparato un piano generale di intervento per le cinque Scuole. Questo piano generale, Plan Rector, prevedeva di completare la riqualificazione dell'intero complesso in non più di cinque anni.

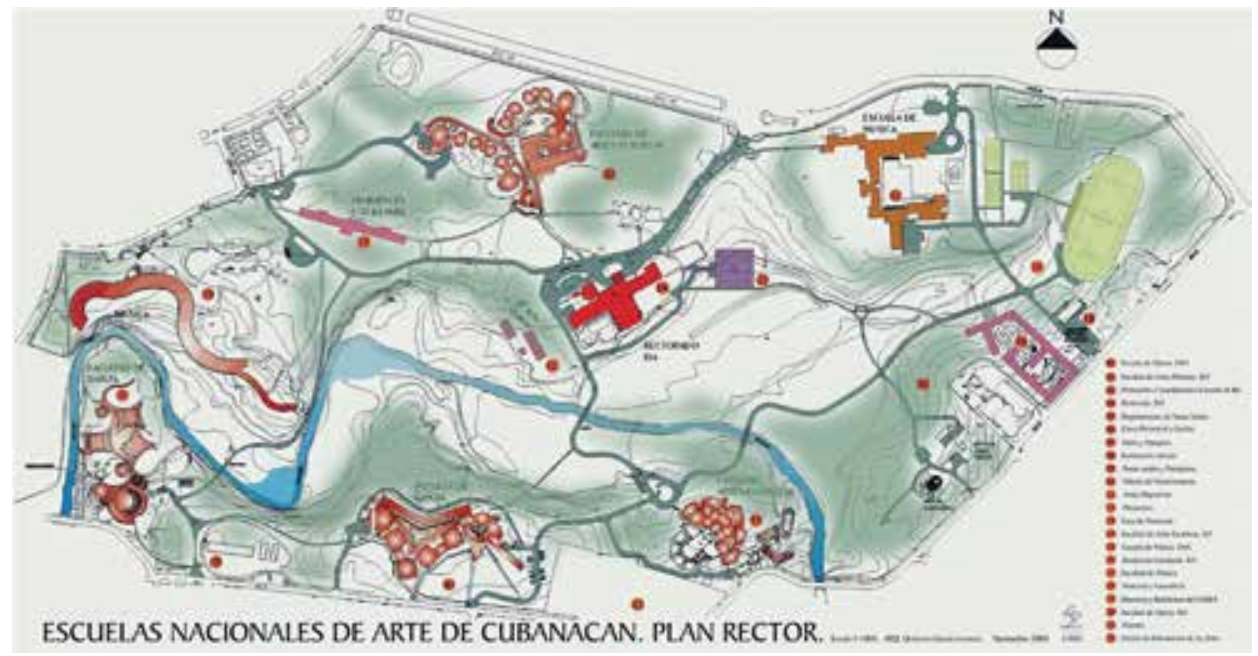

$\mathrm{E}$, anche in ossequio alla riforma didattica sopra citata, aveva i seguenti momenti cardine di intervento:

- Restauro e conservazione del patrimonio edilizio architettonico.

- Ristrutturazione e adeguazione funzionale degli edifici emblematici, conservando l'immagine.

- Trasformazione architettonica e cambio d'uso degli edifici a seconda delle strategie di insegnamento.

- Ricostruzione, completamento e ampliamento degli edifici non finiti, secondo la sua fattibilità.

- Nuove opere per garantire un efficiente funzionamento delle istituzioni.

- Paesaggistica come complemento integratore di tutte le opere dell'insieme.

Va subito notato come, dei sei punti sopra citati, ben la metà implicassero profonde modificazioni alla natura del complesso, in termini di opere nuove e ampliamenti. Viene da chiedersi se si sia ben riflettuto sul rischio che si venisse a snaturare quella idea originaria di un complesso architettonico, funzionale e culturale unico nel suo genere.

Oltre ai punti sopra citati, si produsse una classificazione dei vari interventi, secondo il seguente schema, indicando per ciascun edificio le nuove destinazioni di uso e le integrazioni di uso, senza dimenticare la necessità di residenze per studenti e di edifici dedicati alla manutenzione:

- Edificio originale del Country Club: Rettorato dell'ISA

- Escuelas de Arte: Arti Plastiche, Danza, Ballet, Musica, Arti Sceniche 
- Edifici del sistema Girón: Residenza ISA, Scuola di Musica, Albergo ENA

- Altri servizi: Navi di manutenzione, lavanderia e spogliatoi, magazzini

- Opere nuove: Aree esterne, viali, sentieri, ponti, torrette di vigilanza, illuminazioni e paesaggistica .

E certamente si costruì una metodologia di intervento, a partire dagli studi necessariamente previ, da eseguirsi entro il 2002:

- Rilievi topografici e architettonici

- Perizie tecniche per ogni edificio per quantificare e valutare il deterioramento degli elementi costruttivi e dei materiali di finitura

- Ricerca ingegneristica specializzata quale caratterizzazione di materiali ceramici e altri, indagini strutturali di crepe, cedimenti, corrosione

- Lavori di ricerca sui problemi ambientali microbiologici

- Alberi malati e taglio selettivo

Furono individuate le principali patologie di degrado, classificate in 28 fenomeni, che così si riportano, ricavate da uno dei documenti ufficiali dell'equipe tecnica e in possesso dello scrivente per gentile concessione dell'Arch. Universo Garcìa Lorenzo.

Principali patologie di degrado o problemi costruttivi:

I. Muffa e usura delle lastre in terracotta dei tetti (volte catalane e cupole) verso l'esterno;

2. Umidità, efflorescenze, usura e distacco delle lastre in terracotta dei tetti (volte catalane e cupole) interiormente;

3. Distacco delle lastre in terracotta dei tetti (volte catalane e cupole) verso l'esterno;

4. Fessure longitudinali sull'area centrale inferiore (chiave di volta) dei tetti (volte catalane);

5. Crepe longitudinali sull'area centrale inferiori (chiave di volta) sui tetti (volte catalane);

6. Fessure trasversali sui tetti (volte catalane, interno - esterno ed interno delle cupole);

7. Cedimenti nei pilastri oppure nei contrafforti in mattone delle gallerie (Scuole di Arti Plastiche e Danza), evidenti a causa delle fessure orizzontali nella zona di cambio di sezione dell'elemento e negli appoggi di sostegno delle travi;

8. Distacco della malta o del calcestruzzo dalle travi e dai tenditori del calcestruzzo armato. Corrosione dell'acciaio;

9. Presenza di muffe e/o croste nelle travi e tenditori del calcestruzzo armato.

10. Crepe o fessure nelle travi e tenzonatori del calcestruzzo armato (longitudinali, trasversali, diagonali);

II. Crepe o fessure longitudinali nel punto di unione della cupola con le travi o il muro;

12. Svuotamento o perdita della sezione dei mattoni a vista nelle pareti; 
13. Svuotamento o perdita della sezione e distacco delle lastre in terracotta sulla superficie inferiore delle volte o cupole;

14. Presenza di vernici inadeguate sulle pareti e sui mattoni a vista;

15. Dipinti "artistici" su pareti, cupole e volte, che aggrediscono l'architettura originale

16. Gonfiamenti crepe e distacco degli intonaci in malta si muri in mattone, colonne e altri elementi strutturali con questa finitura (Scuola di Danza);

17. Crepe o fessure sui punti di unione delle pareti con le travi portanti delle volte;

18. Sfondamenti dei pavimenti in lastre di terracotta;

19. Rottura, distacco e anche perdita del pavimento in piastrelle di terracotta;

20. Deterioramento di tutta la carpenteria compresi i lucernari;

21. Deterioramento dei calpestini in legno;

22. Deterioramento degli impianti idrosanitari;

23. Deterioramento degli impianti elettrici;

24. Deterioramento dell'attrezzatura ed altri elementi architettonici;

25. Otturazione delle tubature pluviali e canali di drenaggio;

26. Deterioramento dei rinvestimenti in piastrelle e mobili sanitari;

27. Deterioramento, deficit ed assenza di luminari:

28. Presenze di erba nel corpo del edificio e sui tetti.

Sulla base di perizie tecniche e analisi multidisciplinari si prevedono e intraprendono i seguenti interventi:

- Eliminazione di piante e alberi che danneggiano gli edifici

- Fabbricazione nelle Industrie manifatturiere del Paese dei diversi elementi in ceramica, richiesti a seconda delle caratteristiche definite

- Sostituzione in alluminio laccato con lo stesso design di tutta la carpenteria esterna danneggiata o persa

- Se pavimenti sostituzione di porte e pavimenti in legno di calpestio

- Sostituzione di tutti i mobili sanitari e impianti di evacuazione sanitaria e acqua piovana di drenaggio

- Sostituzione di impianti elettrici e rinnovo del sistema di illuminazione funzionale e ambientale in tutti i settori.

\section{CRONACA DI UN RESTAURO: SECONDA PARTE}

Questa metodologia avrebbe dovuto essere applicata a ciascuna delle cinque Scuole, ma di fatto si iniziò con le uniche due scuole a suo tempo completate, quelle di Ricardo Porro, la Escuela de Arte Plàstica e la Escuela de Danza. Delle ventiquattro persone che ebbero un qualche ruolo tecnico nel restauro delle Scuole di Ricardo Porro, oltre ai tentativi di restauro, dopo, sulle altre Scuole, di fatto Universo Garcia Lorenzo ne rappresentò l'anima, il promotore, il depositario di ogni azione progettuale, tanto che molti, soprattutto in campo internazionale (il cantiere era evidentemente alla attenzione della cultura architettonica internazionale) gli rimproverarono di avere interpretato troppo liberamente il mandato 
affidatogli, qualche volta tralasciando di consultare preventivamente gli autori delle Scuole, limitandosi ad una informazione-post, in cerca di una eventuale asseverazione a cose fatte. E questo fu un secondo motivo di incomprensione fra il grande sforzo che il Governo Cubano mise in piedi per il restauro, affidandosi ai suoi tecnici migliori, e i tre grandi architetti autori.

Anche se va detto che si riconosce una diversa maniera di reagire a questa "incomprensione", tra Ricardo Porro, da una parte, e Roberto Gottardi e Vittorio Garatti dall'altra. Porro per esempio, risulta allo scrivente, avere avuto sempre un atteggiamento disincantato e, in qualche modo, distaccato, da tutta l'operazione. Ricordo, per esempio, che nel maggio del 2006, in una sua conferenza alla sede del Plan Maestro, in Miramar La Habana, affermò, riferendosi al restauro delle sue Scuole: ..... "hagan bien lo que le convenga que hacer .... yo no me ocupo de política ... me ocupo de arte ... me ne frego!" (Ricardo Porro considera la lingua italiana la migliore per pronunciar parolacce, in spagnolo groserias). Nelle sue visite a Cuba durante i primi anni del restauro, si limitò solo a dare dei minimi consigli. Diverse e più determinate furono le lamentazioni di Garatti e Gottardi: essi criticarono soprattutto le disinvolte iniziative di Universo Garcìa, quali la costruzione di garitte o stanzette di vigilanza, alle entrate della Scuola di Danza e a quella di Musica, la costruzione di un sinuoso muro di cinta o un nuovo ponte in cemento armato sul Rio Quibú, tutte pensate e realizzate "alla maniera di", secondo Universo per ragioni di sicurezza e maggior funzionalità di cantiere, secondo i due malpensate e senza un adeguato retroterra di sintesi culturale. Per non parlare della annosa questione delle continue inondazioni dello stesso Rio Quibú nello spazio architettonico della Scuola di Balletto e del tentativo di fermarne l'impeto con un muro in cemento armato.

Lasciando questa doverosa digressione e tornando al cuento de los hechos, così come sono noti allo scrivente, e senza potere entrare, per ora, in temi squisitamente tecnici, va detto che gli studi e i lavori per il restauro delle Scuole di Porro proseguirono per anni, tra molte difficoltà, alcune delle quali in dipendenza delle crisi economiche di cui soffre e ha sofferto la nazione cubana, ma anche in dipendenza delle obiettive difficoltà tecniche legate all'intervento. Ma anche qui alcune considerazioni di carattere generale si impongono: il restauro materico, soprattutto per i rivestimenti ceramici, non fu un intervento "a tappeto", bensì a macchia di leopardo, solo dove la evidente e drammatica situazione di degrado lo necessitava, al punto che alla fine della operazione risultarono evidenti le parti oggetto di intervento da quelle no. I materiali utilizzati per rimpiazzare le piastrelle deteriorate furono importate dal vicino, in tutti i sensi, Venezuela, con le conseguenze tipiche dell'uso nel restauro di materiali di qualità differente. Tanto che lo stesso Universo dovette ammettere, in un recorrido che facemmo insieme nel $201 \mathrm{I}$, che quell'acquisto non era stato dei più felici, e che, a tre anni dalla ultimazione del restauro, le lozas presentavano già problemi. II restauro della Scuola di Arte Plastica e della Scuola di Danza fu terminato nel 2008. Allo stesso tempo le Scuole d'Arti furono dichiarate dal Governo Cubano Monumento Nacional. Nel 2009 all'Arch. Universo García Lorenzo le fu consegnato il Premio Nacional de Restauracíón Cubano. Le due Scuole di Porro sono, alla data attuale, le uniche restaurate.

Già durante i lavori delle Scuole di Ricardo Porro si misero in piedi le strategie di intervento su quella scuola che, tra le tre rimaste, presentava, se così si può dire, meno problematiche: la Scuola di Arte Drammatica di Roberto Gottardi. Qui si trattava di dar mano a una operazione complessa: risultando mai completata, era necessario sia provvedere al restauro e consolidamento della parte esistente, sia al completamento secondo il progetto originale di Gottardi, sia ampliare lo stesso per dar spazio alle nuove esigenze didattiche della riforma ed a una nuova maniera di intendere le fasi della progettazione di un'opera di teatro. 
Figura 5. Scuola di Arti Plastiche, prima del restauro.

Fonte: Arch. Michele Paradiso.
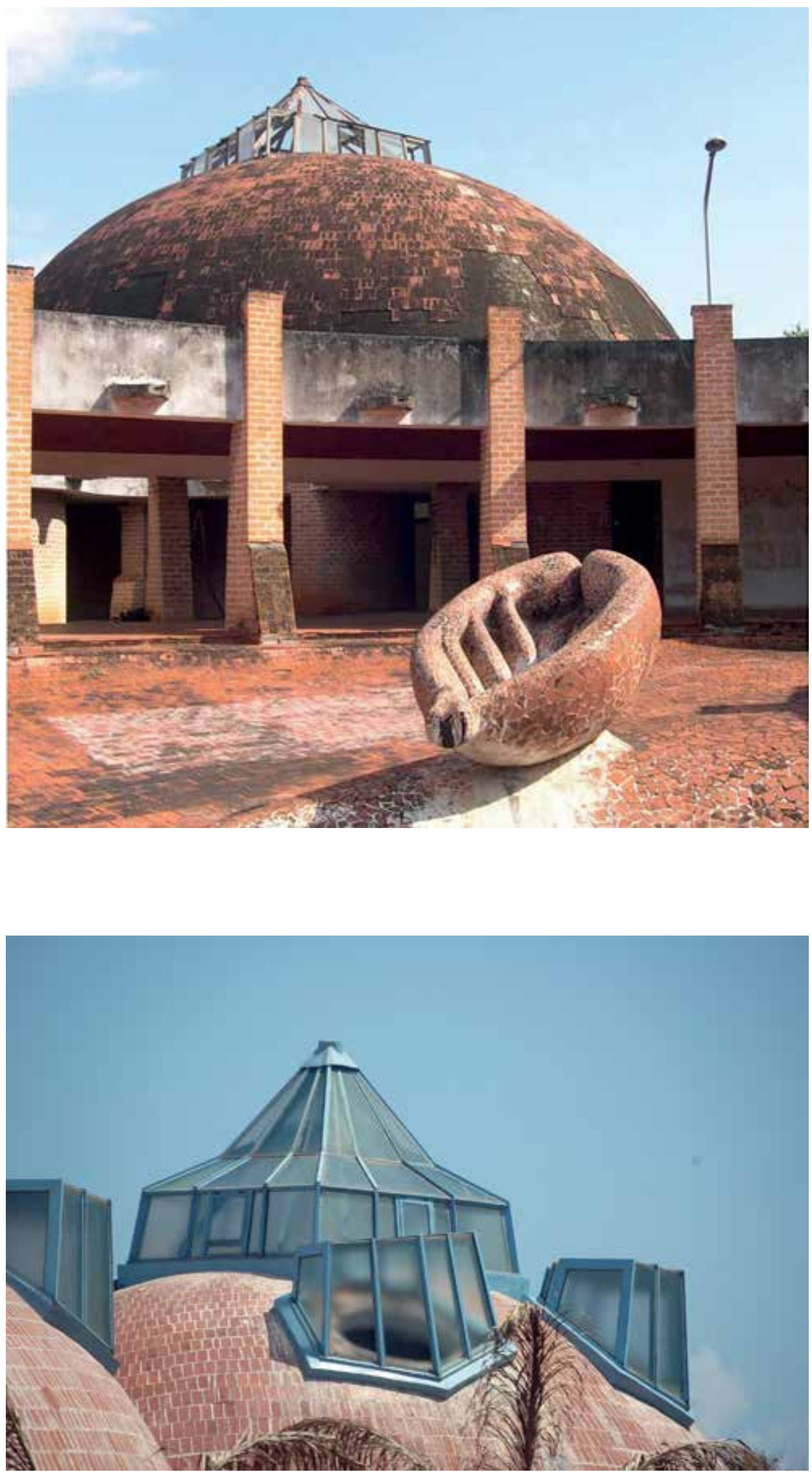

REVISTA M VOL. 11 No.2. JULIO-DICIEMBRE 2014 • FACULTAD DE ARQUITECTURA • UNIVERSIDAD SANTO TOMÁS COLOMBIA M 16 


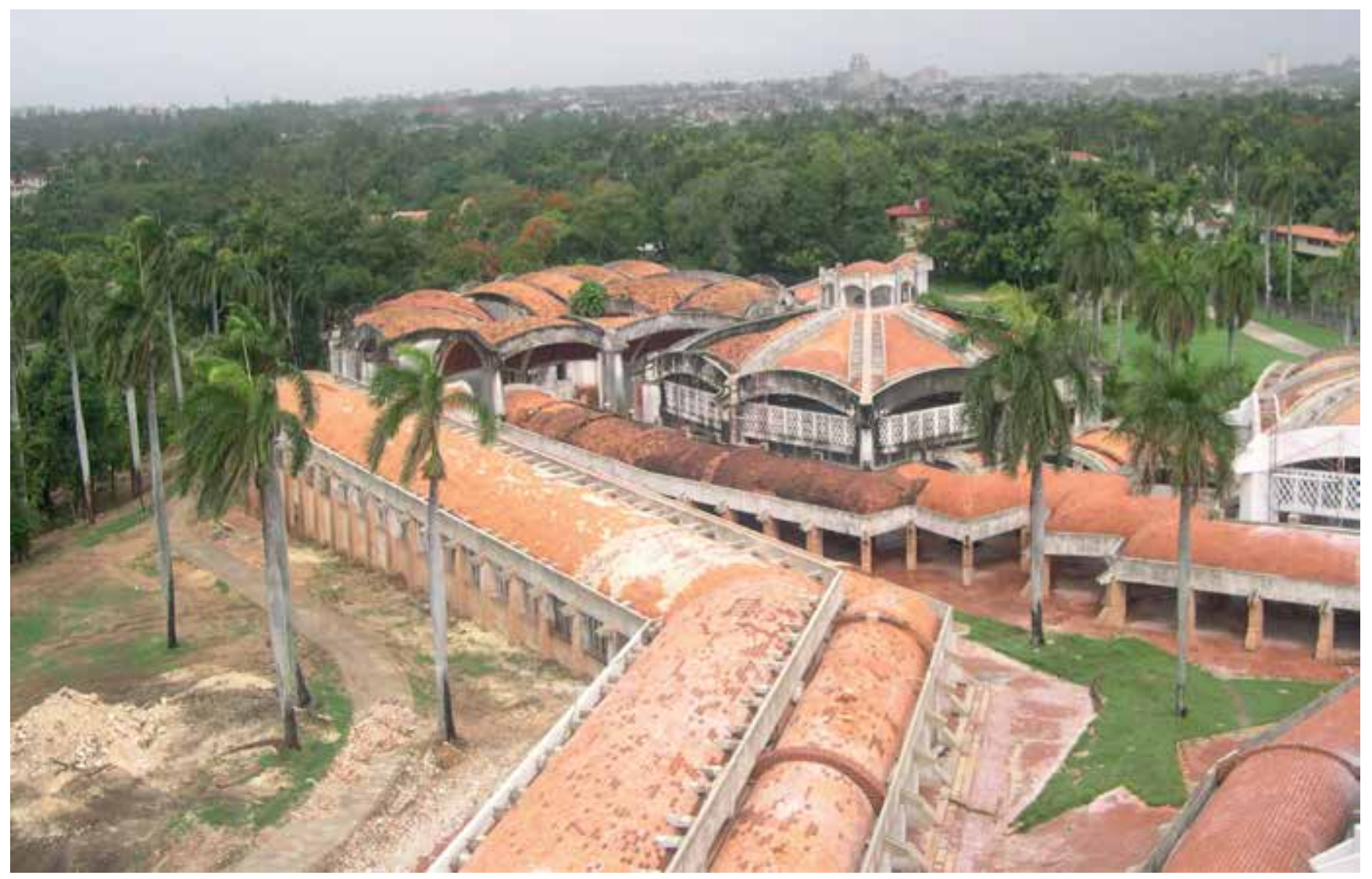

Figura 7. Scuola di Danza, prima del restauro. Fonte: Arch. Michele Paradiso.

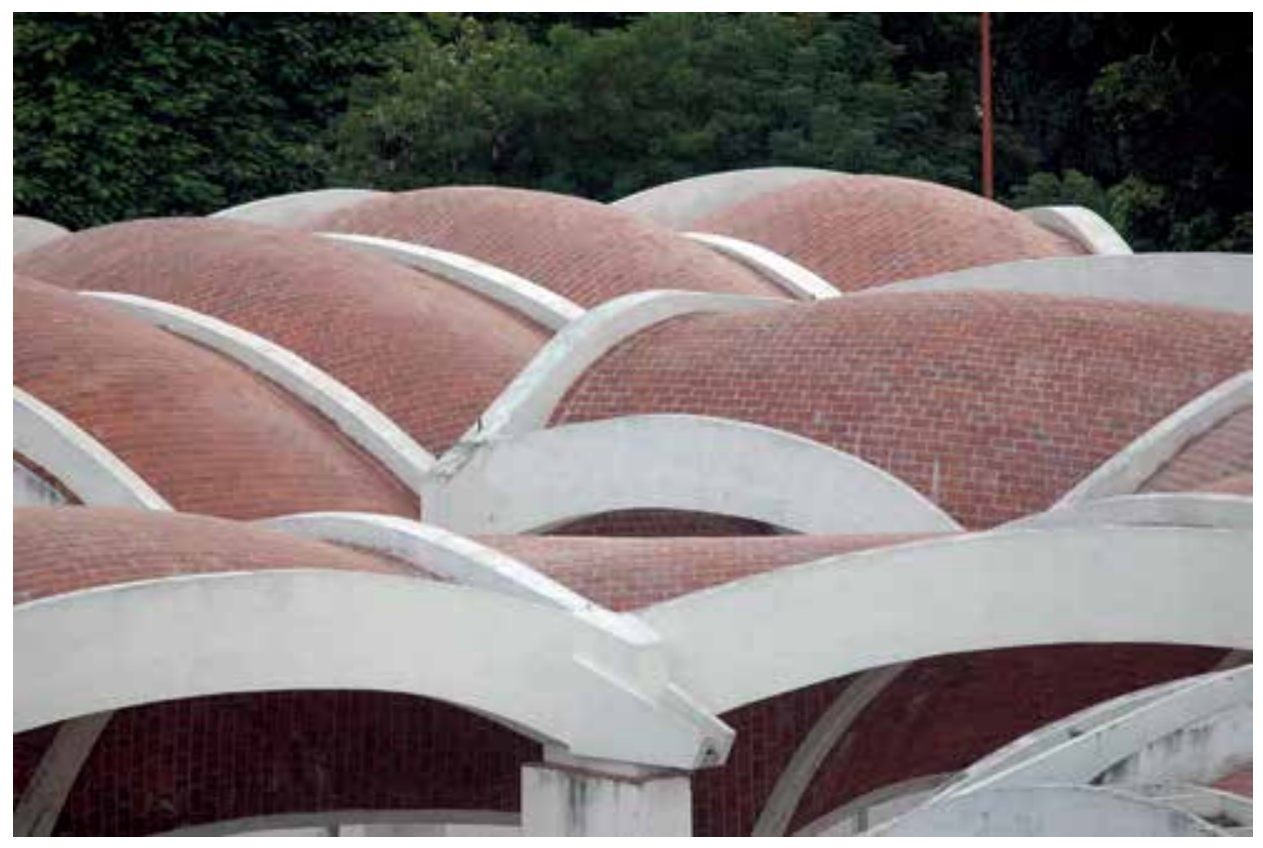

Figura 8. Scuola di Danza, resturata, particolare.

Fonte: Arch. Michele Paradiso.

Per la fase di restauro e consolidamento si applicò la stessa metodologia di diagnosi, affidandosi allo stesso centro CIDEM di Santa Clara. Le indagini diagnostiche riportarono una situazione prevalentemente di degrado materico, sostanzialmente rappresentata da 
intrusioni di formazioni arboree sull'estradosso delle volte catalane, di un forte degrado materico dell'ultimo strato delle piastrelle ceramiche, e di una forte aggressione degli agenti atmosferici che avevano "mangiato" la struttura materica dei mattoni i quali risultarono in alcuni punti corrosi molto di più della stessa malta che li legava.

Questo fenomeno, come è noto, capita quando la qualità dei mattoni cotti in forno non risulta buona ed è fenomeno abbastanza diffuso, ovunque. A un'analisi materica di alcuni campioni fatti analizzare in Italia, risultò una temperatura di cottura di non più di 600 gradi celsius, quando è noto che la cottura deve arrivare ad almeno $800-900$ gradi celsius, pena

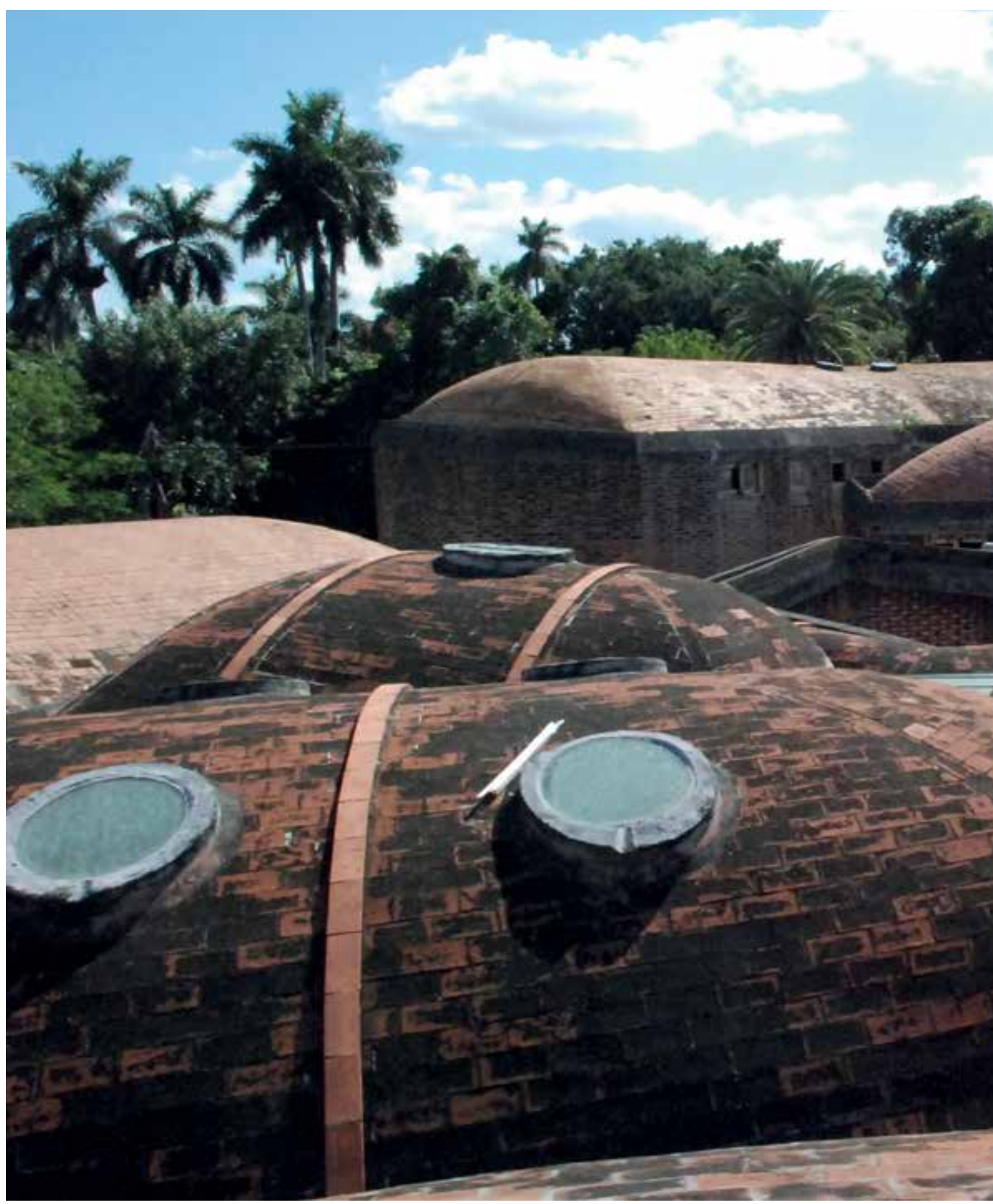


la scarsa resistenza meccanica degli stessi. È questa considerazione aprirebbe un'altra questione e cioè la qualità dei materiali impiegati nella costruzione delle Scuole. Non tutte ebbero, pare, la fortuna di essere costruite con i migliori mattoni. La Scuola di Teatro fu una di queste.

Lo studio del CIDEM portò alla conclusione che alle prime due problematiche si dovesse porre rimedio semplicemente sostituendo completamente l'ultimo strato di piastrelle, permettendo così durante questa fase anche di eliminare le intrusioni arboree. Cosa che effettivamente è stata fatta, iniziando dall'aula in posizione nord-nordovest, e continuando con le aule, in parte, dell'anello esterno.

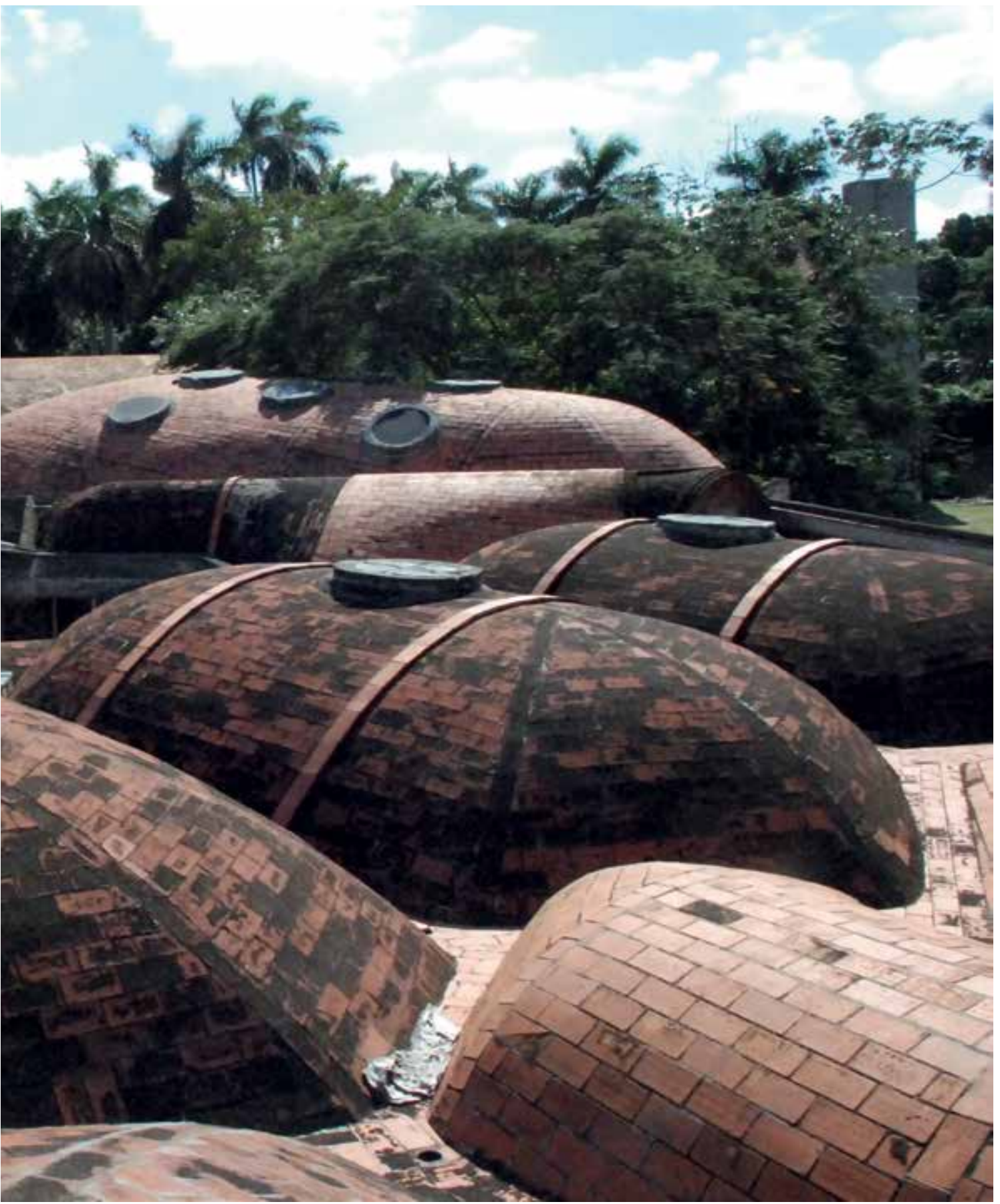

Figura 9. Particolare delle volte catalana in avanzato stato di degrado alla Scuola d'Arti Sceniche.

Fonte: Arch. Michele Paradiso. 
Viceversa nulla fu deciso per le parti di muratura mangiate dal vento e dalle forti piogge, sia perché nel frattempo la storia del restauro delle Scuole ebbe uno stop importante, sia perché sul tema della soluzione da dare si aprì un'altra polemica su come intervenire, tra Roberto Gottardi, che, vivendo a Cuba, aveva evidentemente più occasioni di dialogo con la equipe tecnica, e il Proyectista General. Opinione di quest'ultimo era che, trattandosi di un muro di non più di cinquant'anni di vita, altro non era da fare se non sostituire i mattoni deteriorati con mattoni nuovi, con la semplice tecnica del scuci-cuci. Roberto Gottardi invece, volendo nel restauro mantenere una testimonianza del degrado sofferto in quella interazione architettura-natura, alla base della stessa idea progettuale originaria, desiderava congelare il processo di degrado applicando alla zona ammalorata una sostanza siliconica che ne impedisse l'ulteriore degrado e ne mantenesse l'effetto estetico, quasi che si trattasse di un affresco dipinto sul muro dalla forza della natura. Questione meramente epistemologica e propria di un concetto di restauro filologicamente corretto, non proprio tipico dei mondi latinoamericani.

Quanto alla necessità di adeguare la Scuola di Teatro alle nuove esigenze didattiche della riforma, Roberto Gottardi fu incaricato di redigere un nuovo progetto architettonico, con la richiesta di aumentare il numero delle aule. Essendo il progetto originario in qualche modo a sviluppo chiuso, Gottardi dovette interessarsi a far coesistere la parte di nuova progettazione, di estensione simile alla antecedente, col preesistente. Affrontò il problema con la coscienza che la sua visione dell'architettura e della sua Scuola di Teatro, non poteva mantenersi identica quella di cinquant'anni prima: nascono così volumi più spigolosi, che si raccordano, a fatica, con l'architettura preesistente. Come si sa, lo sforzo progettuale di Roberto Gottardi è anch'esso arenato di fronte alle difficoltà recenti nel proseguire l'opera di restauro de Las ENA.

Un discorso a parte meritano le Scuole di Vittorio Garatti. II maestro, già da tempo pienamente riabilitato, ha viaggiato negli ultimi anni frequentemente a La Habana e accolto sempre col rispetto a lui dovuto. Particolare impulso alla possibilità di restaurare e mettere in funzione la Scuola di Balletto e quella di Musica, si ebbe negli anni in cui il restauro delle sue opere passarono più direttamente nella supervisione del Ministerio de Cultura (2007 e seguenti), avendo come responsabile della riprogettazione Carmen Rosa Bàez. Questi, insieme a José Choy, altro sostenitore da sempre delle necessità di una rivitalizzazione delle Scuole, visitò Roberto a Milano, per prendere i primi accordi sul da farsi, a dimostrazione della attenzione che lo stesso Fidel Castro Ruz aveva verso il problema. Sulla Scuola di Balletto non c'era altro da fare che rimboccarsi le maniche e procedere ad una semi ricostruzione, visto lo stato di vandalismo avanzato e di degrado in cui era stata lasciata, fino al punto che, narra lo stesso Garatti, nel tentativo di rimuovere con una ruspa le macerie accumulatesi nei pasillos e nelle aule interne, vennero incautamente spezzati quasi tutti i tiranti della struttura. Si trattava poi di ripristinare il vecchio ponte sul Rio Quibú, che collegava la Scuola di Balletto a quella di Musica e di mettere in sicurezza, soprattutto il grande anfiteatro circolare, dalle aggressioni delle esondazioni dello stesso Quibú. Sulla prima questione si è già detto di quanto le soluzioni tecniche previste da Universo García non incontrassero il favore di Garatti. Furono anche eseguite attente indagini diagnostiche, da parte della INVES-CONS, Empresa Nacional de Investigaciones Aplicadas, i cui risultati furono pubblicati nel 2008. In quello stesso periodo si discuteva anche sul ripristino della Scuola di Musica, che mancava ancora del grande auditorio. Ma soprattutto Garatti, convinto del "come era e dov'era", anche nella destinazione funzionale, non accettava che parte delle funzioni della sua Scuola fossero attribuite, come si paventava, ad un altro edificio.

Il grande sforzo intrapreso dal Governo Cubano dal 1999, che, malgrado incomprensioni, diffidenze e visioni differenti, era proseguito per dodici anni, terminò di fatto nel $201 \mathrm{I}$, 
quando il Governo Cubano, a causa de la crisi economica internazionale, abbassò gli stanziamenti per il restauro di un $80 \%$. Le opere si fermarono completamente e i fondi non sono bastati nemmeno per una semplice manutenzione dello esistente. All'Arch. Universo García Lorenzo fu dato un altro incarico.

La Scuola di Balletto ha vissuto, in epoca recentissima, e forse vive ancora, un momento di grande visibilità internazionale. Alla fine del 20 I I Carlos Acosta, cubano e primo ballerino del Royal Ballet, convince il nuovo Ministro della Cultura, Rafael Bernal Alemany, a trasformare la Scuola di Balletto nel "Centro Cultural Carlos Acosta", affidando il progetto a Sir Norman Foster. Viene persino firmato un accordo tra il Ministero e la Carlos Acosta International Dance Foundation. L'Arq. Universo Garcìa Lorenzo viene nuovamente incaricato di coordinare, per la parte cubana, le fasi di sviluppo del progetto. L'impresa Atrio incaricata dei lavori. Tutto ciò senza che l'autore dell'opera, Vittorio Garatti, a cui andava riconosciuto per lo meno il diritto d'autore, ne fosse minimamente informato. Incominciano così a diffondersi, da allora, nel mondo internet e nella stampa internazionale, sempre più richiami alla notizia, fino a video nei quali uno dei più grandi architetti contemporanei spiegava la sua idea progettuale. A parte le numerose comunicazioni, riguardo all'avanzamento delle stategie progettuali e alle visite di Sir Norman Foster alle Scuole, del sito della Carlos Acosta International Dance Foundation, valga per tutte citare quanto apparso sulla pagina digitale di bdonline.co.ik, il 2 I giugno 20 I2:

"Foster and Partners has been chosen to design a new art complex for the cuban ballet star Carlos Acosta, that wants to convert the abandoned School of Ballett on the outskirts of Havana. Foster's is working on a redevelopment of the derelict building with its domes roofs, designed in 196I by the italian Vittorio Garatti."

In altre fonti si mostravano al mondo internet plastici del progetto di Foster, simulazioni degli interni del grande anfiteatro, interviste al nuovo progettista e al grande ballerino, con dettagliate descrizioni delle fasi operative del progetto.

La reazione immediata di Vittorio Garatti scatenò un dibattito internazionale, attraverso la rete, sulla opportunità e l'etica di una simile operazione. Sia pur con sfumature diverse, tutti criticarono il metodo, che aveva escluso fin dall'inizio il progettista della Scuola. Piano piano il dibattito fu spostato anche a La Habana, fra i più eminenti intellettuali, architetti, artisti, etc...., fino a un dibattito pubblico che si tenne il I 4 di luglio del 2012 a La Habana, alla presenza del Ministro Bernal, che promise, tra l'altro, di fornire aggiornamenti sullo sviluppo della situazione. Perché si abbia una idea dell'atmosfera di preoccupazione che si viveva nel mondo della cultura architettonica in quei mesi, si riporta in allegato la lettera aperta, a firma di Luisa Marisy e Celma Dìaz, che ulteriormente sollecitava il Ministero di Cultura a una definitiva spiegazione dell'accaduto.

Per sua parte Vittorio Garatti e i suoi estimatori, italiani e non, dettero vita a iniziative di dibattito culturale. Ciononostante non fu possibile che Norman Foster e Vittorio Garatti mai si incontrassero.

II 6 novembre del 2012, la Giuria dell'importante premio culturale italiano "Premio Vittorio De Sica", avendo come Presidente l'eminente critico Gian Luigi Rondi, assegnò ai tre architetti progettisti delle Scuole il primo premio per la sezione "Una vita per l'architettura". II premio fu personalmente consegnato dal Presidente della Repubblica Italiana, Luigi Napolitano, durante una suggestiva cerimonia al Palazzo del Quirinale in Roma, a Roberto 
Gottardi, Vittorio Garatti e Riccardo Porro, invitati nella Capitale Italiana personalmente dal Presidente Napolitano, non solo per dar loro il giusto riconoscimento a una invidiabile percorso professionale e culturale, ma anche, è opinione dello scrivente, per manifestare una particolare attenzione a tutto il problema delle Scuole d'Arte.

II I 3 novembre del 2013 Vittorio Garatti, dopo mesi di silenzio e incomprensioni per i suoi legittimi diritti, invia una lettera all'Arch. Foster invitandolo ad un atteggiamento più rispettoso verso le forme originali della Scuola di balletto e verso il suo autore (in allegato).

Allo stato attuale l'iniziativa di Carlos Acosta pare ferma. Molti dei video nella rete con il progetto di Foster eliminati. II Ministro Bernal sostituito. Nel marzo del 2014 la Fondazione Guayasamin de La Habana ha inaugurato nei suoi locali una mostra antologica dell'opera del Maestro Vittorio Garatti, alla presenza del nuovo Ministro della Cultura di Cuba, Julián González Toledo. Ciò lascia sperare in una ripresa delle iniziative, questa volta veramente partecipate, per il rilancio de Las Escuelas de Artes.

Lo scorso anno, in occasione delle celebrazioni in suo onore che la Casa de Las Tecas Verdes gli ha dedicato, Roberto Gottardi, commentando la storia, irta di difficoltà, delle Scuole, ha definito le stesse, con causticità che solo la sua lunga esperienza professionale e umana gli ha potuto permettere ..... "unas espinas en el culo del arte".

Nella vicenda del restauro delle Scuole, che pare non aver mai fine, sia, per il futuro, di monito la frase di Victor Hugo:

Ci sono due cose in un edificio, il suo uso e la sua bellezza; il suo uso appartiene al proprietario, la sua bellezza a tutti: distruggerlo è dunque oltrepassare i propri diritti.

\section{REFERENZE}

ENIA (MICONS), CIDEM (Universidad Central "Marta Abreu” de Las Villas). (2006). Informe Proyecto de Investigación, Instituto Superior de Arte: Escuela de Arte Plástica. Santa Clara, Cuba.

ENIA (MICONS), CIDEM (Universidad Central "Marta Abreu” de Las Villas). (2007). Informe Proyecto de Investigación, Instituto Superior de Arte: Escuela de Arte Escénica. Santa Clara,Cuba.

ENIA (MICONS). (2008). Informe Escuela de Música. Diagnóstico, Análisis y Evaluación Estructural. La Habana, Cuba.

ENIA (MICONS). (2008). Informe técnico, Instituto Superior de Arte: Escuela de Ballet. La Habana, Cuba.

Gulli, R. (2006). La Costruzione Coesiva. Venezia: Marsilio edizioni.

Loomis, J. (2012). Revolution of Forms, Cuba's Fortgotten Art School. New York: Princeton Architectural Press.

Paradiso, M. (2004). II Restauro de las Escuelas de Arte a Cuba. Incontro con Roberto Gottardi. Progettare Architettura-Citta'-Territorio, Anno III (I8). Ed. Tecniche Nuove Spa, Milano. 
Paradiso, M. (2006). Las grietas del pasillo de la Escuela de arte Plastica de Ricardo Porro. Análisis estructural y interpretación: una contribución al debate. Acta de la III Bienal de Arquitectura de La Habana, La Habana, mayo 2006.

Paradiso, M. (20I I). II recupero de Las escuelas de Arte de Cubanacàn: una missione impossibile?. Cuba. Scuole Nazionali D’Arte (pp. I56-162). Milano: Skira editore.

Paradiso, M. (2006, noviembre). Análisis estructural de la escuela de Arte Plástica de Ricardo Porro en La Habana. En Proceedings of 7th International Symposium of Structures, Geotechnics and Constructions Materials, Santa Clara. Cuba.

Paradiso, M. (2006, septiembre). La recuperación arquitectónica y estructural de las Escuelas de Arte de Cubanacàn en La Habana, Cuba. En Proceedings of I I th International Seminar on FORUM UNESCO/UNIVERSITY and HERITAGE, Florencia, Italia.

Paradiso, M.(2005). II Restauro de las Escuelas Nacionales de Arte a La Habana, Cuba. Costruire in laterizio. Anno XVIII, Nov.-Dic. Faenza Editrice.

Paradiso, M., \& Simoni, M. (noviembre, 2008) Estudio estàtico de la Escuela de Arte Escènica de Roberto Gottardi en las ENA de Cubanacán: un punto de vista italiano para su rehabilitación. En Proceedings of 7th International Symposium of Structures, Geotechnics and Constructions Materials", Santa Clara, Cuba.

Pérez, L., García, U. y otros. (2003). Informe de investigaciòn y proyecto de restauración de las Escuelas Nacional de Arte de Cubanacán. La Habana, Cuba. 